\title{
eJRIEPS
}

Ejournal de la recherche sur l'intervention en éducation physique et sport

$23 \mid 2011$

Varia

\section{Analyser l'activité décisionnelle de joueurs de football en situation d'entraînement pour développer un modèle de joueur virtuel}

Cyril Bossard, Gilles Kermarrec, Camille De Keukelaere, Denis Pasco et Jacques Tisseau

\section{OpenEdition}

Édition électronique

URL : http://journals.openedition.org/ejrieps/4580

DOI : 10.4000/ejrieps.4580

ISSN : 2105-0821

Éditeur

ELLIADD

Référence électronique

Cyril Bossard, Gilles Kermarrec, Camille De Keukelaere, Denis Pasco et Jacques Tisseau, « Analyser l'activité décisionnelle de joueurs de football en situation d'entraînement pour développer un modèle de joueur virtuel », eJRIEPS [En ligne], 23 | 2011, mis en ligne le 01 avril 2011, consulté le 21 février 2020. URL : http://journals.openedition.org/ejrieps/4580 ; DOI : 10.4000/ejrieps.4580

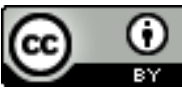

La revue eJRIEPS est mise à disposition selon les termes de la Creative Commons Attribution 4.0 International License. 
Cyril Bossard*, Gilles Kermarrec ${ }^{* *}$, Camille De Keukelaere*, Denis Pasco** et Jacques Tisseau*

* Laboratoire d'Informatique des Systèmes Complexes (LISyC EA 3883). Centre Européen de Réalité Virtuelle 25 rue claude chappe 29280 Plouzané

** UFR Sport et EP de Brest. Université de Bretagne Occidentale

Résumé

Notre étude s'est attachée à la description de l'activité décisionnelle de joueurs de football en situation de contre-attaque. Cette activité décisionnelle a été appréhendée à l'aide du cadre théorique de la NDM en situation aménagée à des fins d'étude. Des données comportementales ont été enregistrées auprès de douze joueurs de football, puis complétées par des données verbales recueillies lors d'un entretien d'autoconfrontation. Les données ont été analysées en 6 étapes: (a) la retranscription des données, (b) la sélection et l'identification des unités significatives, (c) le découpage du déroulement de l'activité en situations vécues, (d) l'identification des situations et des schémas, (e) la modélisation de la dynamique de l'activité décisionnelle, et (f) la validité de l'analyse. Les résultats ont permis d'identifier 16 schémas caractéristiques de l'activité décisionnelle des joueurs de football en situation de contraintes temporelles. Ces schémas constituent des structures d'arrière-plans articulant des composantes perceptives et cognitives, et qui facilitent la reconnaissance rapide de situations pendant la contre-attaque. La discussion de ces résultats pointe l'aspect dynamique de l'activité décisionnelle. L'application de ces résultats à un modèle de joueur de football virtuel est ensuite présentée et des perspectives d'évolution sont proposées.

\section{Introduction}

L'activité décisionnelle de joueurs de sports collectifs a souvent été étudiée en isolant ses composantes perceptives et cognitives. Cette approche dite computationnelle de l'activité décisionnelle est restée longtemps dominante en psychologie du sport (Bossard \& Kermarrec, sous presse). La prise de décision y est considérée comme un processus à part entière du système de traitement de l'information, située entre l'extraction des informations de l'environnement et l'exécution de l'action (Schmidt, \& Lee, 2005). 
Assez récemment, certains auteurs (Araùjo, Davids, \& Hristovski, 2006 ; Lenzen, Theunissen, \& Cloes, 2009 ; Mouchet, \& Bouthier, 2006) ont souligné les limites d'un recours exclusif au modèle du traitement de l'information pour étudier l'activité décisionnelle dans le domaine des activités sportives.

Dans le cadre d'une approche ergonomique de l'activité, la décision peut être appréhendée au cours de l'action et étudiée dans sa globalité. L'objet d'étude est alors élargi à la notion d' "activité » (Bedny, Karwowski, \& Bedny, 2001) en considérant la décision comme indissociable de l'action. D'un point de vue épistémologique, ces investigations que nous qualifierons de «naturalistes " considèrent la décision comme un processus d'adaptation à la situation courante (Ross, Shafer, \& Klein, 2006). L'activité décisionnelle est décrite à travers le couplage dynamique entre un acteur et une situation. Dans cet article, nous proposons d'utiliser l'approche Naturalistic Decision Making (NDMpour «prise de décision naturelle », notre traduction) pour analyser l'activité décisionnelle de joueurs de football dans une situation dynamique typique en sports collectifs : la contreattaque. L'intérêt de cette approche théorique, habituellement exploitée dans les situations de travail, réside dans son intention de mettre en œuvre une analyse globale (englobante) et contextualisée des processus perceptifs et cognitifs associés à l'action. La proximité entre ces situations professionnelles ou de formation et les situations de sports collectifs a déjà été soulignée (Fiore \& Salas, 2006 ; Sève et al., 2009), mais peu d'études empiriques ont été réalisées en sport à l'aide de l'approche NDM (Macquet, 2009).

\section{Le cadre théorique de la NDM}

Le courant NDM s'est donné comme objectif d'améliorer les systèmes d'aide à la décision dans le domaine militaire mais aussi dans l'industrie nucléaire et le secteur de l'aviation civile. II étudie la façon dont des experts, travaillant seuls ou en groupe dans des environnements dynamiques et incertains, identifient et évaluent des situations, prennent des décisions et exécutent des actions dont les conséquences sont significatives pour eux et pour leur environnement (Lipshitz, Klein, Orasanu, \& Salas, 2001) Les situations de sports collectifs sont des situations dynamiques par excellence. Dans une équipe, les rôles sont évolutifs notamment dans les situations de contre- attaques qui imposent au joueur de prendre des décisions pertinentes sous fortes contraintes temporelles. Une contre-attaque est une situation de jeu offensive effectuée le plus rapidement possible dès la récupération du ballon afin de prendre de vitesse le repli défensif adverse. Cette situation de jeu est particulièrement intéressante à étudier car elle permet de caractériser et de modéliser l'activité décisionnelle de joueurs exposés à de fortes contraintes spatio- 
temporelles. Au sein de l'approche NDM, le modèle Recognition-Primed Decision (RPD pour "décision fondée sur la reconnaissance ", notre traduction) développé par Klein (1997 ; 2008) est particulièrement adapté pour analyser le couplage acteurs - situations.

\section{1. Le modèle Recognition-Primed Decision}

Le modèle RPD permet d'expliquer les décisions d'experts prises sous fortes pressions temporelles et imprégnées d'enjeux (Hoffman, \& Lintern, 2006). Klein et Brezovic (1986) réfutent l'idée selon laquelle les individus confrontés aux situations dynamiques fondent leurs choix sur la base d'un calcul rationnel ou d'une analyse exhaustive des utilités (théorie des jeux ou théorie formelle de la décision). Pour Ross et al. (2006), les experts font principalement appel à leur expérience pour prendre des décisions. L'accent est porté sur l'activité décisionnelle comprise comme englobante et complexe, i.e. un phénomène "macro-cognitif » émergent (Klein, Ross, Moon, Klein, Hoffman, \& Hollnagel, 2003), où les processus perceptif et cognitif sont inextricablement liés. Un expert confronté à une situation dynamique est capable de reconnaître la typicalité de la situation et d'y associer une réponse adaptée ou acceptable.

Plus précisément, pour Klein (1997, 2008), l'évaluation de la situation en cours d'action reposerait sur la reconnaissance de configurations spatio-temporelles significatives. Le choix d'une solution optimale passe par la reconnaissance implicite de patterns, significatifs pour l'action. La situation s'impose à l'individu qui la " découvre » et dont l'expertise se traduit par une reconnaissance finalisée et rapide de cette situation. Au cours d'une situation dynamique, ce processus de reconnaissance repose sur la mobilisation d'un "package cognitif» (Ross et al., 2006). Ce package associe quatre types de variables secondaires : les attentes, les indices pertinents, les cours d'actions typiques issus de l'expérience, et les buts plausibles. Le " coup d'œil » de l'expert en cours d'action, consiste en une mise en correspondance implicite entre les indices pertinents perçus et des structures fonctionnelles disponibles en mémoire. Certains auteurs ont proposé de considérer que ces quatre variables étaient associées au sein d'une structure cognitive, qualifiée de schéma (Lipshitz et al., 2001).

Complémentairement, le modèle RPD proposé par Klein $(1997$; 2008) distingue trois modalités de reconnaissance utilisées par les experts face à une situation dynamique : une reconnaissance simple, un diagnostic de la situation, et une évaluation d'un cours d'action. La première modalité peut être qualifiée de processus réactif grâce auquel l'expert reconnait une situation déjà rencontrée et y associe directement une réponse adéquate. La seconde modalité relève d'un processus de diagnostic en comparant des indices pertinents de la situation rencontrée avec plusieurs situations 
similaires précédemment vécues, afin de choisir parmi des réponses connues et mettre en œuvre une action appropriée. Dans la troisième modalité, l'expert élabore une solution inédite dans le cours de l'action et l'évalue par un processus de simulation mentale. Ce processus lui permet d'imaginer comment son action pourrait s'intégrer à la situation courante.

Le modèle RPD a été exploité dans diverses situations dynamiques issues du domaine du travail (pour une revue voir Ross et al., 2006). Dans le domaine du sport, le modèle RPD a été validé dans des conditions expérimentales au handball (Johnson \& Raab, 2003) et dans des conditions réelles d'entraînement et de match respectivement au hockey sur glace et au volleyball (Bossard, De Keukelaere, Cormier, Pasco, \& Kermarrec, 2010 ; Macquet, 2009).

Johnson et Raab (2003) ont présenté des vidéos de séquence de jeu de handball à un panel de joueurs de niveaux variés. Après une présentation du film de 10 secondes, les sujets devaient nommer le plus rapidement possible la première décision qui leur venait intuitivement à l'esprit en se mettant à la place du joueur porteur de balle. Dans un second temps, ils donnaient le maximum d'options qu'ils pouvaient imaginer face à cette même situation. Enfin, parmi l'ensemble des réponses données, les sujets étaient invités à évaluer la meilleure d'entre elles pour cette situation. Les résultats ont montré que les joueurs de handball prenaient des décisions pertinentes dès le premier cours d'action considéré. De plus, quand les experts abandonnaient leur première intuition en faveur d'une autre décision générée à la suite, la qualité du cours d'action subséquent était plus faible que le premier naturellement considéré.

Macquet (2009) a analysé l'activité décisionnelle de joueurs experts au cours d'un match de volley-ball. Les résultats ont montré que dans $85 \%$ des situations vécues durant un match, les joueurs de volleyball investissaient principalement la première modalité du modèle RPD pour prendre des décisions («simple match »), contre 12,8\% des situations pour la seconde et $2,7 \%$ pour la troisième. Bossard et al. (2010) ont confirmés par la suite que la pression temporelle exercée sur les joueurs de hockey sur glace en situation de contre-attaque les contraignait à mobiliser prioritairement cette première modalité. Complémentairement, cette dernière investigation menée en situation naturelle a montré que les catégories constitutives du «package cognitif » du hockeyeur expert recouvraient les quatre variables initiales du modèle RPD et pouvaient être complétées par une cinquième variable appelée connaissance (Bossard et al., 2010).

Pour reconnaître une situation comme typique et y répondre rapidement, les experts disposeraient de structures mnémoniques permettant de conserver des potentiels 
d'action significatifs et efficaces. Cette hypothèse renvoie à l'idée que les personnes mémorisent et organisent des informations d'expériences passées sous une forme abstraite, i.e. des schémas. Certains tenants de l'approche NDM avancent que la reconnaissance d'une situation résulte de l'activation de schémas et/ou de scripts (Flin, Slaven, \& Stewart, 1996 ; Randel, Pugh, \& Reed, 1996 ; Piegorsch, Watkins, Piegorsch, Reininger, Corwin, \& Valois, 2006).

\section{2. La théorie des schémas}

Le concept de schéma a été proposé en psychologie cognitive ergonomique pour étudier conjointement le rôle des structures cognitives impliquées dans un processus adaptatif et le rôle des contextes qui affectent leur mise en œuvre (Anderson, Matessa, \& Lebiere, 1997). Les schémas sont des structures abstraites issues de l'expérience. Ils sont réutilisés pour prendre des décisions rapides face à des situations nouvelles, similaires ou identiques (Rumelhart, 1980). Les schémas permettent aux experts de prendre des décisions en catégorisant de manière efficiente la situation comme un pattern entier (Federico, 1995).

La notion de schéma est proche de la notion piagétienne de schème (Piaget, 1947). Pour Vergnaud (1990), le schème est « l'organisation invariante de la conduite pour une classe de situations données » (p. 136). Chalandon (2007) relève la proximité entre les éléments constitutifs d'un schème et le "package cognitif » permettant l'évaluation de la situation au sein du modèle RPD (buts, actions, indices pertinents, attentes). Cette proximité l'amène à considérer le fonctionnement du modèle RPD comme un processus d'évocation d'un schème. Les schémas englobent les objets, les partenaires sociaux, les positions dans l'espace et les événements. Le schéma permet d'interpréter une situation dans sa globalité. Exploitant le modèle RPD en volley-ball, Macquet (2009) montre le processus d'évaluation globale de la situation. Les volleyeurs experts mobilisaient un processus de décision qui s'appuyait à la fois sur l'évaluation de la situation et sur le choix d'une action. Plus précisément, les résultats ont montré que dans $84,4 \%$ des situations, l'activité décisionnelle des joueurs se basait sur la première modalité du modèle RPD. L'utilisation de l'une ou l'autre des modalités de prise de décision était dépendante du temps alloué et du caractère plus ou moins connue de la situation rencontrée. Plus la situation vécue était proche, similaire d'une situation déjà connue, plus la décision pouvait être rapide. L'étude de Macquet (2009) suggère ainsi que les experts utiliseraient leur expérience pour agir.

L'identification des schémas permet une description des éléments de référence au sein d'un domaine d'expertise. D'autres concepts habituellement considérés comme 
proches du concept de schéma permettent de penser l'organisation en mémoire de ces structures cognitives: frame ou cadre (Minsky, 1975) et script ou scénario (Schank, \& Abelson, 1977). Si le cadre représente une structure de données liée à un contexte, le scénario décrit une chronologie, une séquence événementielle.

La théorie des schémas appliquée par Bossard et al. (2010) dans une étude récente sur l'activité décisionnelle de hockeyeurs experts lors de contre-attaques met en avant le rôle et l'adaptation de ces structures d'arrière-plan. Les auteurs montrent l'activation et l'adaptation permanente des schémas en fonction du contexte. Pour choisir la bonne action à réaliser, la principale condition nécessaire est alors la mise en correspondance entre certains invariants contextuels de la situation vécue et des invariants d'arrière-plan qui permettent d'agir : les schémas. Complémentairement, quand les membres d'un groupe (ou d'une équipe sportive) partagent des expériences, ils construisent des schémas similaires, ce qui les conduit à répondre de façon semblable dans des situations prototypiques au sein du domaine de référence (Piegorsch et al., 2006).

\section{3. Présupposés et objectif de l'étude}

Pour étudier l'activité décisionnelle des joueurs dans une situation dynamique, la contre-attaque en football, nous avons exploité le modèle RPD et le concept de schéma. Notre travail s'est appuyé sur quatre présupposés : 1) l'activité décisionnelle est un processus de reconnaissance qui relève d'une association entre des éléments présents en arrière plan et des indices pertinents relevés dans le contexte ; 2) l'activité décisionnelle en situation dynamique peut être décrite, commentée et explicitée de façon continue par l'acteur confronté aux traces de cette activité (les changements de significations du contexte pour l'acteur permettant d'analyser son activité comme une succession de situations vécues) ; 3) chaque situation vécue traduit l'activation d'un « schéma », faisant référence pour percevoir et décider en cours d'action, et 4) l'analyse de l'enchainement des situations devrait permettre une description diachronique de l'expertise en situation de contre-attaque et la mise en évidence de scénarii préférentiels .

\section{Méthode}

\subsection{Participants}

L'étude a été menée en collaboration avec douze joueurs de football volontaires issus de la même équipe, de niveau national dans leur catégorie (championnat national U17), et appartenant à un centre de formation d'un club de football professionnel. Un contrat moral concernant leur anonymat a été passé dans le cadre de cette étude. Afin de 
garantir cette relative confidentialité, les joueurs ont été nommés à l'aide de pseudonymes: Charles, Jordan, Benjamin, Thibault et Paul étaient des défenseurs; Gautier, Kévin et Michel occupaient les postes de milieux de terrain ; Bastien, Gaétan, Flavien et Benoît étaient des attaquants. Les joueurs sélectionnés étaient, en moyenne, âgés de 15,8 ans (écart type : 0,38) et pratiquaient le football en compétition en moyenne depuis 10 ans. Leur activité décisionnelle a été analysée au cours d'une situation d'étude privilégiée.

\section{2. Situation d'étude privilégiée}

Pour cette étude nous avons choisi d'analyser l'activité des joueurs lors d'une situation d'entraînement. Cette situation visait selon l'entraîneur "l'amélioration de la progression rapide de la balle grâce à la coordination des attaquants lors de la contre attaque ». Plus précisément, la situation choisie était une situation d'opposition à trois contre trois sur un demi-terrain de football à 11 où les équipes avaient pour objectif de marquer un but le plus rapidement possible. L'équipe analysée était l'équipe qui attaquait sur la grande cible (but standard). L'autre équipe attaquait sur une petite cible large de 1,5 mètre. Au départ de la situation, les équipes s'affrontaient dans une zone proche de la ligne médiane du terrain. Nous avons ajouté une règle contraignante à l'équipe non analysée : " le joueur qui perd le ballon est pénalisé, il doit faire le tour d'un plot disposé à 10 mètres derrière la petite cible ". Cette règle a permis à l'équipe analysée d'être temporairement en situation de surnombre. Pour que cette situation d'étude privilégiée soit significative, "réaliste ", les entraîneurs ont insisté sur la nécessité de mettre en place des conditions qui permettent aux joueurs de passer de la phase défensive (récupération du ballon) à la phase offensive (contre-attaque).

Cette situation d'entraînement constituait pour nous une situation d'étude privilégiée. En effet, des études antérieures ont montré que l'activité collective d'équipes sportives reposait principalement sur des coordinations locales entre 2 ou 3 joueurs (Bourbousson \& Sève, sous presse) et sur des constructions circonstancielles issues du contexte (Mouchet \& Bouthier, 2006). Ces travaux ont pointé l'intérêt d'études relatives à l'évolution de l'activité individuelle - collective. C'est pour nous centrer sur la dynamique de l'activité que nous avons choisi de travailler à l'aide d'une situation « réduite » et partiellement prédéfinie. 


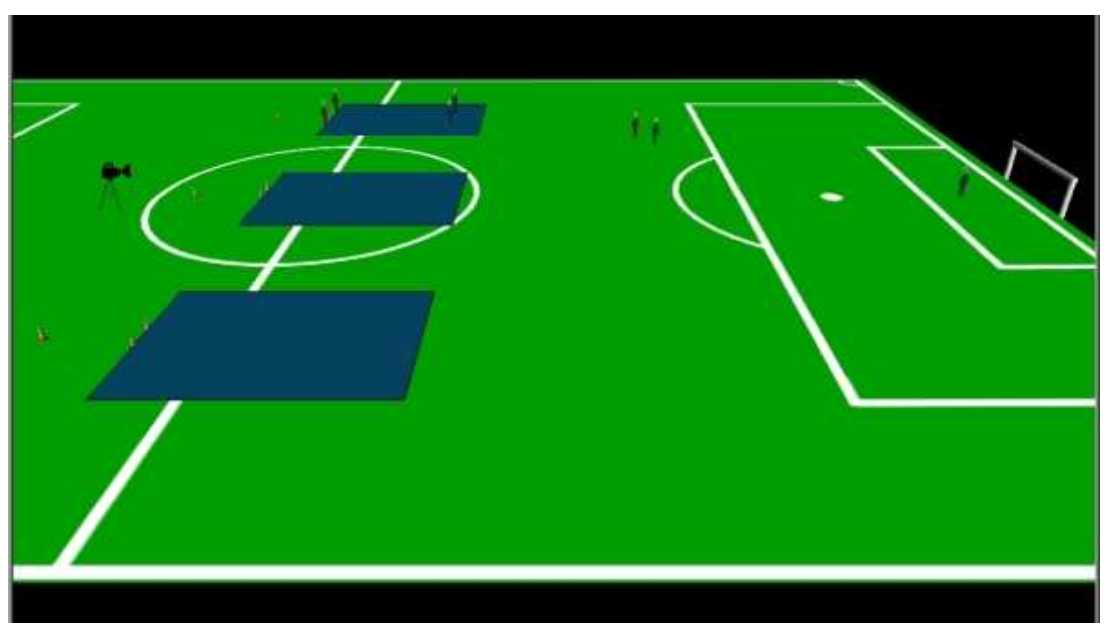

Figure 1. Représentation 3D de la situation d'étude privilégiée

Cette situation d'étude favorisant les contre-attaques a été construite en collaboration avec plusieurs entraîneurs du centre de formation. Elle a été testée 2 fois par un groupe de joueurs et un entraîneur différents. Cette exploration a permis de questionner les joueurs et les entraîneurs sur la crédibilité de la situation au regard des contre-attaques vécues lors de compétitions. Des allers-retours entre pratiquants, entraîneurs, et chercheurs ont permis d'affiner les consignes à donner aux participants, et d'ajuster certaines caractéristiques de la situation (distance des plots, taille des zones, règles supplémentaires).

\section{3. Recueil des données}

Les douze joueurs étudiés ont été séparés en quatre équipes de trois joueurs. Chaque joueur a effectué cinq passages dans la situation d'étude. La composition des équipes a été réalisée par l'entraîneur. Ce dernier les a organisées en cherchant à réunir des joueurs habitués à évoluer régulièrement ensemble (entraînements et matchs). L'activité décisionnelle des joueurs a été étudiée à partir de 60 situations de contreattaques. Deux types de données ont été recueillis: (a) des données d'observation relatives à l'activité des joueurs en phases de contre-attaque et (b) des verbalisations provoquées lors d'un entretien d'autoconfrontation.

Les données d'observation ont été recueillies grâce à l'enregistrement audiovisuel des comportements lors de la situation d'étude privilégiée. L'intégralité des passages a été enregistrée à l'aide d'une caméra vidéo numérique placée sur pied fixe et positionnée en arrière et sur le côté du terrain (Cf. Figure 1). Nous avons opté pour un plan fixe, large, cadrant le demi-terrain où se déroulait la situation d'étude. Ce dispositif a permis d'enregistrer en continu les actions des trois joueurs évoluant sur le terrain et leurs adversaires. 
Tous les joueurs ont participé à la phase de verbalisation. Les données de verbalisation ont été recueillies au cours d'entretiens d'autoconfrontation individuels menés à l'issue de la séance d'entraînement. Ces données ont été enregistrées à l'aide d'un magnétophone et d'une caméra filmant les participants. Lors de ces entretiens, le chercheur et le joueur ont visionné ensemble la cassette vidéo des passages du joueur dans la situation d'étude. Au cours de cette visualisation, le joueur a été invité à décrire et à commenter son activité. Les relances ont porté essentiellement sur les actions qui étaient significatives (et par conséquent décrites et explicitées) par les joueurs au cours de la situation de contre-attaque et sur des événements pour lesquels le chercheur souhaitait obtenir des informations complémentaires. Tous les entretiens ont été réalisés par le même chercheur qui a déjà réalisé de tels entretiens au cours d'une précédente étude (Bossard et al., 2010).

\section{4. Traitement des données}

Soixante situations de contre-attaque ont donné lieu à une analyse des données (cinq par équipe de trois joueurs et d'une durée qui variait entre 10 et 15 secondes). Chaque film vidéo a été visionné de façon à répertorier le plus précisément possible les actions des protagonistes d'une façon neutre, c'est-à-dire sans inférence relative à leurs intentions. L'intérêt de disposer des comportements en vis-à-vis des verbatim est de fournir des indices à l'analyste pour appréhender la logique de l'acteur. Pour chaque situation de contre-attaque, les communications verbales des joueurs et du chercheur au cours des entretiens ont été intégralement retranscrites. Les données ont été analysées en 6 étapes : a) la retranscription, b) la sélection et le codage des unités significatives, c) le découpage du déroulement de l'activité en situations vécues, d) l'identification des schémas, e) la modélisation de l'enchainement des schémas en cours d'action, et f) la validité de l'analyse

\section{4. 1. La retranscription des données}

Cette étape consiste à préparer les données recueillies en vue des analyses de contenu successives. Pour chaque joueur, des protocoles à trois volets ont été réalisés en respectant le décours temporel de la situation d'étude (Tableau 1). La première colonne référence la situation étudiée afin de faciliter l'analyse pour le chercheur. Dans une deuxième colonne, le contexte objectif est décrit par le chercheur au travers des changements de rôles du joueur et d'une description des événements en évitant toute interprétation. Dans une troisième colonne, les verbalisations obtenues lors des entretiens d'autoconfrontation ont été retranscrites et placées en vis-à-vis des comportements observés. 


\section{4. 2. La sélection et le codage des unités significatives}

Cette étape a consisté à sélectionner et identifier des unités significatives, c'est-àdire des comportements observés et des passages ou phrases du discours du joueur qui renseignaient sur son activité décisionnelle. Deux critères ont été successivement utilisés pour sélectionner les unités significatives au cours de l'analyse de contenu : 1) la verbalisation était compatible avec le comportement décrit en vis-à-vis ; 2) la verbalisation désignait l'activité en relation directe avec la situation de contre-attaque, c'est-à-dire qu'elle renseigne sur ce que le joueur fait, perçoit, ressent, ou pense effectivement dans cette situation de jeu. Pour coder les unités significatives sélectionnées, le système de catégories défini par le modèle RPD (Klein, 1997 ; 2008) et récemment complété par Bossard et al. (2010) a été utilisé. Pour chaque unité significative, nous avons attribué un code (en lettre majuscule) qui renvoyait aux cinq composants du modèle RPD : les buts plausibles $(B)$, les cours d'actions typiques $(A)$, les indices perçus comme significatifs (I), les attentes $(A R)$ et les connaissances $(C)$ (Tableau 1).

2. 4. 3. Le découpage du déroulement de l'activité en situations vécues

Cette troisième étape a consisté à découper le flux de l'activité du joueur pour identifier et distinguer les situations successives vécues par (du point de vue de) chaque athlète lors des situations de contre-attaque. Nous avons repéré des ruptures dans le discours du joueur sur son activité à l'aide de critères de forme et de fond. Dans la mesure où on admet que le discours est une forme de restitution de l'action, ces connecteurs peuvent constituer certains indices de la temporalité vécue.

Les connecteurs, en tant que marque langagière, ont été une aide précieuse pour repérer ces ruptures (alors que, quand, puisque, afin de, pour). Ces connecteurs peuvent être considérés comme des traces d'un remaniement du couple joueur-situation (CaronPargue, \& Caron, 1989). Les critères de forme du discours ont ainsi permis de repérer la clôture (donc) et l'ouverture (ici, ensuite, là) d'une situation ou la réorientation de l'activité du joueur vers un nouvel objet (puis, dès que, quand). Repérer ces connecteurs permettait de repérer l'enchaînement des situations vécues et des schémas activés.

Les critères de fond ont le plus souvent été des unités significatives relatives aux indices pertinents (de nouvelles informations contextuelles étaient prises en compte par le joueur), aux buts (apparition d'une nouvelle intention) ou aux actions qui pouvaient constituer une finalisation de la situation vécue (Je tire au but). Ces unités significatives qui marquent l'ouverture ou la fermeture d'une situation étaient particulièrement saillantes et déterminantes dans le déroulement de l'activité du joueur. 
Ces ruptures successives (identifiées à partir du fond et de la forme du discours) dans le déroulement de l'activité du joueur ont permis de découper l'activité du joueur en situations successives. Par hypothèse, ces situations vécues par le joueur renvoyaient au contexte perçu comme significatif pour lui au travers d'un schéma activé. En regroupant par similitudes l'ensemble des situations vécues, l'analyse a permis d'identifier des situations typiques de la contre-attaque au football et les schémas activés par les joueurs dans notre situation d'étude. Le Tableau 1 illustre les étapes 1, 2, 3 de notre analyse des données.

Tableau 1. Catégorisation des unités significatives et découpage de l'activité en situations vécues.

\begin{tabular}{|c|c|c|}
\hline Situation d'étude & Données d'observation & Données d'autoconfrontation \\
\hline \multicolumn{3}{|l|}{$3^{\mathrm{eme}}$ contre-attaque } \\
\hline Equipe 2 Michel & & Situation vécue 1 \\
\hline $1^{\prime}$ & $\begin{array}{l}\text { Michel presse son adversaire } \\
\text { direct, il récupère la balle, se } \\
\text { retourne, et lève la tête. }\end{array}$ & $\begin{array}{l}\text { Je récupère la balle }(A) \text {, je lève la tête }(A) \text {, et } \\
\text { je regarde directement où sont mes } 2 \\
\text { partenaires }(A) \text {, je conduis la balle }(A)\end{array}$ \\
\hline $5^{\prime}$ & $\begin{array}{l}\text { Michel fait une longue passe à } \\
\text { Benoît dans l'axe, }\end{array}$ & $\begin{array}{l}\text { Situation vécue } 2 \\
\text { Ici, je la donne à Benoît (A), il a libéré de } \\
\text { l'espace en embarquant le défenseur avec lui } \\
\text { (I), je lui mets la balle en profondeur (A). }\end{array}$ \\
\hline \multirow[t]{2}{*}{8} & $\begin{array}{l}\text { Michel continue sa course sur } \\
\text { le côté droit, repique dans } \\
\text { l'axe, }\end{array}$ & $\begin{array}{l}\text { Situation vécue } 3 \\
\text { Là, après je vais au deuxième poteau (A), j'ai } \\
\text { vu Flavien revenir dans ma zone au centre (I), } \\
\text { j'attends que Benoît me fasse la passe (AR), } \\
\text { le défenseur qui revient la touche du bout du } \\
\text { pied (I). }\end{array}$ \\
\hline & & Situation vécue 4 \\
\hline $12^{\prime}$ & $\begin{array}{l}\text { Michel récupère le ballon, } \\
\text { rentre dans la surface, pique } \\
\text { le ballon avec extérieur du } \\
\text { pied droit, marque un but }\end{array}$ & $\begin{array}{l}\text { Là, je la récupère quand même }(A) \text {, je me } \\
\text { mets dans la position de frapper (A), le } \\
\text { défenseur me tacle (I), je l'évite }(A) \text { et je mets } \\
\text { une pichenette par-dessus le gardien }(A) \text {. Là } \\
\text { j'ai vu que le gardien s'avance vers moi et se }\end{array}$ \\
\hline $18^{\prime}$ & & $\begin{array}{l}\text { couche (I), je pique la balle (A) et ça rentre } \\
\text { (B). }\end{array}$ \\
\hline
\end{tabular}




\section{4. 4. L'identification des schémas}

A la quatrième étape de notre analyse, nous avons utilisé une catégorisation empirique des situations vécues (Strauss, \& Corbin, 1998). Nous avons procédé à des regroupements des différentes situations vécues de la même façon par différents joueurs. Parfois, l'identification d'un moment précis de la contre-attaque (début ou fin) nous a aidé à regrouper plusieurs situations vécues. Le titre conceptuel n'était défini qu'en fin d'analyse. Au regard de nos présupposés théoriques, il représentait un schéma c'est-àdire, une structure d'arrière-plan mobilisée au cours de situations différentes mais similaires.

\section{4. 5. La modélisation de la dynamique de l'activité décisionnelle}

Cette étape consistait à modéliser l'enchainement des schémas identifiés chez les joueurs de football afin de rendre compte de la dynamique de l'activité décisionnelle en situation de contre-attaque. Nous avons observé que certains schémas étaient souvent associés, l'un après l'autre, alors que d'autres n'étaient jamais enchaînés. Dans un tableau à double entrée, nous avons alors indiqué les relations existantes (ou non) entre les schémas et le poids de cette relation pour les 7 contre-attaques les plus efficaces (i.e. rapides, fluides et qui aboutissent à une frappe cadrée). A titre d'illustration, quand deux schémas ont été enchaînés 8 fois (quel que soit le joueur), le poids de leur relation était 8. Nous avons ensuite utilisé un logiciel de représentation graphique hiérarchique et orientée (Graph Visualization Software version 2.16.12) permettant de rendre compte de ces relations entre les schémas. Cette approche de la représentation graphique est proche de la plupart des programmes de graphes hiérarchiques (Sugiyama, Tagawa, \& Toda, 1981). Les schémas ont été représentés par des nœuds et la relation entre deux schémas par un arc. Le poids donné à la relation entre deux schémas est représenté par la distance (longueur de l'arc) entre deux nœuds. Le logiciel a ensuite restitué un graphe dont l'agencement tient compte de l'ensemble de ces paramètres (voir Gansner, Koutsofios, North, \& Vo (1993) pour une explication des algorithmes de dot sous-jacents à la modélisation).

\section{4. 6. La validité de l'analyse}

Différents principes ont été appliqués pour assurer la crédibilité des données (Strauss, \& Corbin, 1998). Selon le principe d'exclusivité mutuelle, les unités d'analyse (situations vécues) ne peuvent appartenir qu'à une seule catégorie à la fois. Toutes les unités d'analyse doivent également pouvoir être classées dans une même catégorie (principe d'exhaustivité). Enfin, le regroupement proposé doit être univoque (principe de fidélité). Pour le processus de catégorisation empirique, nous considérons que le modèle 
élaboré traduit bien la diversité du phénomène (i.e., la diversité des schémas activés en phase de contre-attaque) lorsque toutes les données de nouveaux joueurs peuvent être assimilées et associées aux données précédentes sans nécessiter la création d'une nouvelle catégorie au sein du modèle (phénomène de saturation d'après Piegorsch et al. (2006)). Enfin, le codage théorique et le regroupement empirique ont été validés par une procédure de "triangulation » entre trois chercheurs familiers de l'objet d'étude. Les catégories sont proposées par le premier chercheur, les deux autres effectuent individuellement une relecture attentive. En cas de désaccord, les trois chercheurs construisent une proposition commune.

\section{Résultats}

Les résultats obtenus sont présentés en trois points : (a) les catégories constitutives du modèle RPD, (b) les situations vécues et les schémas activés, et (c) la modélisation de l'activité décisionnelle des joueurs en situation de contre-attaque.

\section{1. Le modèle RPD et ses constituants}

Le travail d'identification et de codage des unités significatives (US) nous a permis de dénombrer 1216 unités significatives permettant de décrire l'activité décisionnelle de 12 joueurs en situation de contre-attaque. Conformément au modèle RPD et ses constituants, l'analyse des données par catégorisation théorique fait apparaître que ces unités se répartissent en fonction des 5 catégories théoriques attendues : but (B), action (A), indices pertinents (I), attente (AR) et connaissance (C).

- 548 US $(45,1 \%)$ renvoyaient à des indices perçus par les joueurs. La prise en compte d'éléments pertinents du contexte pour les joueurs concernait: (1) leurs partenaires ("Je vois que Paul prend la balle»), (2) leurs adversaires («Je vois le défenseur entre les 2 »), et (3) des informations sur eux-mêmes ( Je suis bien placé »).

- 393 US $(32,3 \%)$ renvoyaient aux actions réalisées par le joueur (i.e. ce que fait le joueur en situation). Certaines d'entre-elles étaient verbalisées directement par le sujet tandis que d'autres ont été codées au regard des comportements observés. Les actions étaient réalisées en référence à ses propres déplacements ( "Je rentre sur l'aile "), à ceux des adversaires ("Je me mets avec eux»), et partenaires ( JJe l'appelle avec la voix pour lui dire que je suis derrière »), et enfin à la récupération de la balle (" Je la prends »).

- 126 US (10,4\%) correspondaient aux connaissances des joueurs mobilisées pendant l'action. Ces unités ont montré que les experts en situation convoquaient des connaissances singulières qui concernaient : leur propre activité ( "je suis trop statique, je n'ai pas d'élan pour bien la taper »), les adversaires (“ je sais qu'il va vite le défenseur, 
qu'il court plus vite que moi »), les partenaires («techniquement il est meilleur, Paul voit mieux le jeu que moi »), la situation de contre-attaque ( il faut aller vite »), la conception du jeu au football ( “c'est plus joli si Gaétan finit l'action, c'est plus collectif !»), ou encore les règles du jeu ( « je peux pas aller loin, sinon je suis hors-jeu »).

- 102 US (8,4\%) correspondaient aux intentions ou buts des joueurs pendant l'action. Ces unités traduisaient la préoccupation du joueur (« Je veux aller vite devant pour frapper »; “ mon idée c'est de l'éliminer ») en réaction à la reconnaissance d'une situation type.

- 47 US (3,9\%) renvoyaient aux attentes des joueurs relatives à l'action en cours. Ces US représentaient les hypothèses faites par le joueur sur l'évolution de la situation ( «je pense qu'il va me la mettre sans essayer de dribbler»). Ces attentes pouvaient être satisfaites ou non.

Finalement, nous avons obtenu une liste d'unités significatives dont le classement était univoque. A l'issue de cette analyse, l'ensemble des 1216 unités significatives sélectionnées ont été classées dans l'une ou l'autre des catégories. Ce classement a été confirmé par deux autres chercheurs. La répartition des unités significatives par joueur et par catégorie est présentée dans le Tableau 2.

Tableau 2. Répartition des unités significatives par sujet et par catégorie.

\begin{tabular}{lcccccccc}
\hline Joueurs & Poste & Equipe & Buts & Connaissances & $\begin{array}{c}\text { Indices } \\
\text { Pertinents }\end{array}$ & Actions & Attentes & Total \\
\hline Gautier & M & 1 & 6 & 22 & 77 & 43 & 5 & $\mathbf{1 5 3}$ \\
Bastien & A & 1 & 8 & 9 & 45 & 40 & 1 & $\mathbf{1 0 3}$ \\
Gaétan & A & 1 & 10 & 15 & 78 & 37 & 5 & $\mathbf{1 4 5}$ \\
Charles & D & 2 & 10 & 8 & 39 & 33 & 2 & $\mathbf{9 2}$ \\
Jordan & D & 2 & 7 & 10 & 33 & 30 & 3 & $\mathbf{9 3}$ \\
Kevin & M & 2 & 9 & 4 & 35 & 29 & 3 & $\mathbf{8 0}$ \\
Flavien & A & 3 & 11 & 19 & 26 & 31 & 3 & $\mathbf{9 0}$ \\
Benoit & A & 3 & 11 & 11 & 73 & 31 & 6 & $\mathbf{1 3 2}$ \\
Michel & M & 3 & 6 & 6 & 34 & 34 & 2 & $\mathbf{8 2}$ \\
Benjamin & D & 4 & 8 & 4 & 39 & 29 & 9 & $\mathbf{8 9}$ \\
\hline
\end{tabular}




\begin{tabular}{lcccccccc}
\hline Thibault & $\mathrm{D}$ & 4 & 10 & 16 & 28 & 31 & 8 & $\mathbf{9 3}$ \\
Paul & $\mathrm{D}$ & 4 & 6 & 2 & 31 & 25 & 0 & $\mathbf{6 4}$ \\
\hline Total & -- & -- & $\mathbf{1 0 2}$ & $\mathbf{1 2 6}$ & $\mathbf{5 4 8}$ & $\mathbf{3 9 3}$ & $\mathbf{4 7}$ & $\mathbf{1 2 1 6}$ \\
\hline$\%$ & -- & -- & $\mathbf{8 . 3 9}$ & $\mathbf{1 0 . 3 6}$ & $\mathbf{4 5 . 0 7}$ & $\mathbf{3 2 . 3 2}$ & $\mathbf{3 . 8 7}$ & $\mathbf{1 0 0}$ \\
\hline
\end{tabular}

Note $: A=$ attaquant $; D=$ défenseur $; M=$ milieu

3. 2. Identification des situations vécues et catégorisation des schémas

Le découpage du déroulement de l'activité de chaque joueur, en prenant en compte des indices relatifs à la forme et au sens du discours, nous a permis d'identifier 180 situations vécues pour l'ensemble des douze joueurs lors de la situation d'étude. Par un processus de catégorisation empirique nous avons regroupé ces situations dans seize "schémas ». Le Tableau 3 répertorie le nombre de "schémas " activés pendant les 60 situations de contre-attaque par tous les joueurs. Certains schémas peuvent être considérés comme typiques de l'expertise en situation de contre-attaque car ils sont activés par différents joueurs dans diverses situations. L'activité décisionnelle des joueurs de football en situation de contre-attaque s'organise alors autour de 16 schémas.

\section{3. La modélisation de l'activité décisionnelle}

Les résultats obtenus à l'aide du logiciel de représentation graphique Graphviz ont permis de restituer le déroulement des situations de contre-attaque telles qu'elles ont été vécues par les joueurs de football. La modélisation obtenue a permis de proposer une représentation diachronique de l'activité décisionnelle, c'est-à-dire une description des " enchaînements préférentiels de schémas ou scénarii privilégiés " pour les 7 situations de contre-attaque les plus efficaces (voir représentation graphique: Figure 2). Cette procédure de modélisation peut être répétée pour l'ensemble des contre-attaques et des participants, pour chacun des 12 sujets ou encore par équipe, en fonction des besoins de la modélisation.

Le logiciel Graphviz permet de visualiser 2 « scénarii » particuliers selon le déroulement de la situation de contre-attaque (axe $\mathrm{X}$ ) et l'évolution du statut du joueur (axe Y). Les schémas sont disposés de gauche à droite et suivent quatre "phases " de la contreattaque : (1) Transition (schémas 1 et 2), (2) Continuité (schémas de 3 à 9), (3) Recherche de rupture (schémas de 10 à 14), et (4) Finition (schémas 15 et 16). Sur l'axe $Y$, les schémas sont disposés en fonction du rôle du joueur : porteur de balle (PB) ou non porteur de balle (NPB). Les schémas $1,4,8,9,12,14$, et 16 représentent le joueur en 
possession de la balle. Les schémas 2, 3, 6, 7, 10, 13, et 15 représentent le joueur qui évolue sans ballon.

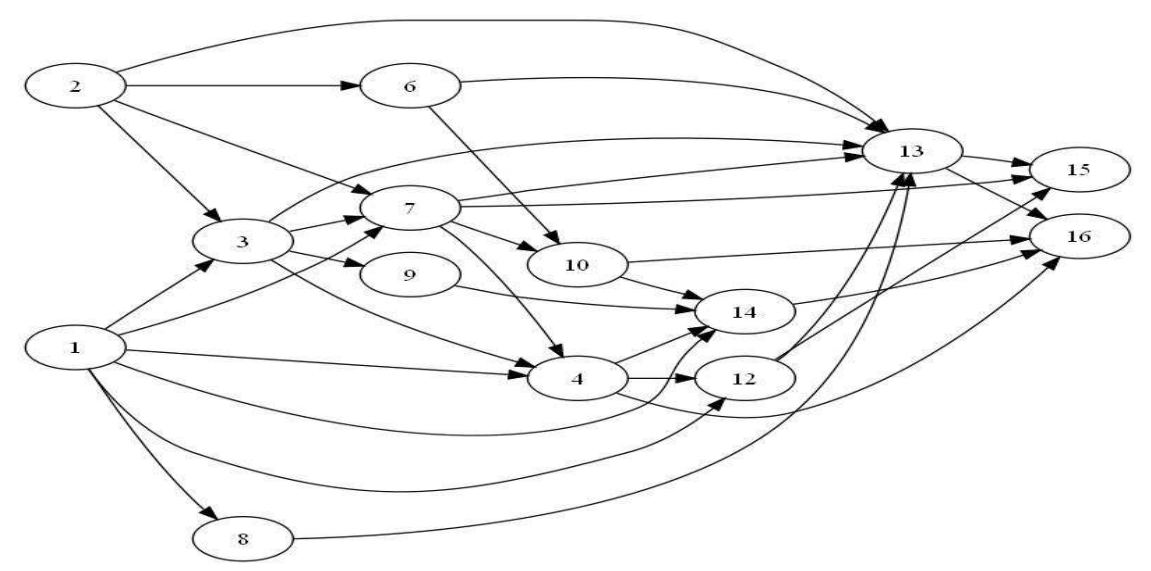

Figure 2. Représentation graphique des schémas activés par les joueurs de football pour les sept contre-attaques les plus efficaces. 
Tableau 3. Nombre d'occurrences des 16 schémas par joueur durant les 60 situations de contre-attaque.

\begin{tabular}{|c|c|c|c|c|c|c|c|c|c|c|c|c|c|}
\hline & & Equipe & & & quipe 2 & & & quipe 3 & & & quipe 4 & & Total \\
\hline Schémas & $\begin{array}{l}\text { Gautier } \\
\text { (M) }\end{array}$ & $\begin{array}{l}\text { Bastien } \\
\text { (A) }\end{array}$ & $\begin{array}{l}\text { Gaétan } \\
\text { (A) }\end{array}$ & $\begin{array}{l}\text { Charles } \\
\text { (D) }\end{array}$ & $\begin{array}{l}\text { Jordan } \\
\text { (D) }\end{array}$ & $\begin{array}{l}\text { Kevin } \\
\text { (M) }\end{array}$ & $\begin{array}{l}\text { Flavien } \\
\text { (A) }\end{array}$ & $\begin{array}{l}\text { Benoit } \\
\text { (A) }\end{array}$ & $\begin{array}{l}\text { Michel } \\
\text { (M) }\end{array}$ & $\begin{array}{l}\text { Benjamin } \\
\text { (D) }\end{array}$ & $\begin{array}{l}\text { Thibault } \\
\text { (D) }\end{array}$ & $\begin{array}{l}\text { Paul } \\
\text { (D) }\end{array}$ & \\
\hline 1. Intercepter le ballon & 2 & 4 & 4 & 2 & - & 2 & - & 2 & 1 & - & - & - & 17 \\
\hline $\begin{array}{l}\text { 2. Observer, analyser, anticiper } \\
\text { l'évolution de la situation }\end{array}$ & 2 & 1 & - & 1 & - & - & 2 & 2 & 1 & 2 & - & - & 11 \\
\hline $\begin{array}{l}\text { 3. S'engager vite vers l'avant pour } \\
\text { profiter de la récupération du ballon }\end{array}$ & - & - & 3 & - & - & 2 & 2 & 1 & - & - & - & - & 8 \\
\hline $\begin{array}{l}\text { 4. Aller vite vers l'avant avec le } \\
\text { ballon tout en étant attentif au jeu }\end{array}$ & 1 & - & - & 2 & 3 & 1 & 1 & 1 & - & - & 2 & 2 & 13 \\
\hline $\begin{array}{l}\text { 5. Proposer son aide à un } \\
\text { partenaire pour conserver le ballon }\end{array}$ & 2 & 1 & 2 & - & - & - & - & - & - & - & - & 1 & 6 \\
\hline $\begin{array}{l}\text { 6. Créer de l'espace pour permettre } \\
\text { à des partenaires de s'engager vers } \\
\text { l'avant }\end{array}$ & - & - & - & 3 & - & 1 & 1 & 1 & - & 4 & - & - & 10 \\
\hline $\begin{array}{l}\text { 7. Se déplacer par rapport à ses } \\
\text { partenaires dans l'espace de jeu } \\
\text { pour proposer une solution de } \\
\text { progression vers la cible }\end{array}$ & 2 & 2 & 2 & 1 & 3 & - & 1 & 2 & 2 & 1 & 3 & 1 & 20 \\
\hline $\begin{array}{l}\text { 8. Choisir une solution de passe } \\
\text { pour assurer la continuité du jeu }\end{array}$ & 3 & 1 & 2 & - & - & 1 & - & - & 2 & - & - & 1 & 10 \\
\hline $\begin{array}{l}\text { 9. Attendre le soutien des } \\
\text { partenaires }\end{array}$ & 1 & - & - & - & - & - & 1 & - & - & - & - & - & 2 \\
\hline $\begin{array}{l}\text { 10. Se déplacer par rapport au hors- } \\
\text { jeu, à l'adversaire et rechercher la } \\
\text { rupture }\end{array}$ & 2 & 1 & 1 & 3 & - & - & - & 1 & - & 2 & 1 & - & 11 \\
\hline $\begin{array}{l}\text { 11. Eliminer en dribblant pour } \\
\text { progresser vers la cible }\end{array}$ & 2 & 1 & - & - & - & - & - & - & - & - & 1 & 2 & 6 \\
\hline 12. Fixer-passer pour progresser & 1 & 1 & . & 1 & 1 & - & 1 & 1 & 1 & - & - & 1 & 8 \\
\hline
\end{tabular}


vers la cible

\begin{tabular}{|c|c|c|c|c|c|c|c|c|c|c|c|c|c|}
\hline $\begin{array}{l}\text { 13. Se placer ou se déplacer pour } \\
\text { recevoir un centre ou une passe } \\
\text { décisive }\end{array}$ & 2 & 1 & 3 & 2 & 3 & 1 & 4 & 2 & 2 & 2 & - & 1 & 23 \\
\hline $\begin{array}{l}\text { 14. Passer pour mettre le partenaire } \\
\text { en situation favorable de frappe }\end{array}$ & 2 & 2 & - & 1 & 1 & 2 & 1 & 1 & 1 & - & 2 & - & 13 \\
\hline $\begin{array}{l}\text { 15. Suivre l'action du partenaire } \\
\text { pour une reprise }\end{array}$ & 1 & - & 1 & - & - & - & - & 2 & 1 & - & - & 1 & 6 \\
\hline 16. Chercher à tirer/frapper au but & - & 1 & 2 & 2 & 2 & 1 & 2 & - & 2 & - & 2 & 2 & 16 \\
\hline
\end{tabular}




\section{Discussion}

Les résultats de notre étude sont discutés selon trois axes, relatifs (a) au contenu des éléments du schéma activé lors du couplage joueur-situation, (b) à la dynamique, l'évolution de l'activité en cours d'action et (c) à la modélisation d'un joueur de football virtuel.

4. 1. Les éléments pris en compte par les joueurs de sports collectifs pour décider

A l'aide du modèle RPD nous avons codé l'activité décisionnelle des joueurs selon 4 variables : des attentes, des buts, des actions, et des indices pertinents. Nos résultats ont montré l'implication d'un cinquième élément chez ces joueurs de football pour décider en situation de contre-attaque: les connaissances. Ce résultat est conforme à celui obtenu en Hockey sur glace dans une étude également réalisée sur des contre - attaques extraites d'une situation de match à l'entraînement (Bossard et al., 2010).

L'analyse du contenu de ces 5 variables a permis d'en préciser les contenus. Les attentes des joueurs concernaient principalement des informations sur l'évolution de la situation vécue telles que s'attendre à une action précise de son partenaire. Les buts correspondaient aux intentions du joueur pendant l'action comme chercher à fixer un défenseur pour l'éliminer. Les actions réalisées par les joueurs se référaient à leurs propres déplacements, ceux des adversaires et des partenaires, et enfin à la récupération du ballon. Les indices perçus par les joueurs concernaient les actions des partenaires et des adversaires, et plus particulièrement leurs déplacements. Enfin, les connaissances exprimées par les joueurs se référaient au joueur lui-même, aux points forts ou faibles des adversaires et des partenaires, à la situation de contre-attaque, à la conception du jeu au football, ou encore aux règles du jeu ou règles d'actions individuelles et collectives. Nos résultats ont ainsi permis de spécifier, dans le contexte particulier d'une situation d'étude favorisant les contre-attaques, les éléments considérés comme significatifs par les joueurs de football pour décider.

Récemment, plusieurs études qualitatives visant à décrire l'activité décisionnelle individuelle en sport collectif à partir du couplage entre le joueur et la situation ont été menées (Bossard et al., 2010 ; Lenzen et al., 2009 ; Macquet, 2009). Leurs résultats ont pointé un ensemble d'éléments pour décider au regard de la situation vécue par le joueur. A la lumière de nos propres résultats, nous observons des similitudes et des divergences avec le contenu des catégories proposées par ces études. A partir d'une démarche d'analyse inductive, Lenzen et al. (2009) ont montré que l'activité décisionnelle des joueurs de Handball se fondait sur la convocation de 4 types d'éléments: (a) perception (visuelle, auditive, tactile, proprioceptive); (b) attentes (intentions des partenaires et 
adversaires); (c) connaissances (concepts, caractéristiques des adversaires et partenaires, expérience) et (d) contexte (score, difficulté du match). Macquet (2009) a également montré la pertinence du modèle RPD pour étudier l'activité décisionnelle des joueurs experts en volley-ball à partir des quatre composants initiaux. Les résultats de Bossard et al. (2010) ont pointé l'intérêt d'intégrer un cinquième composant au modèle RPD : les connaissances.

Le contenu des unités significatives relatives aux « indices pertinents » pour nous et Macquet (2009) ou «perception » pour Lenzen et al. (2009) renvoient aux mêmes objets. Pour les trois études, le contenu fait référence à (a) des actions propres au joueur, (b) des actions des partenaires, (c) des actions des adversaires, et (d) la trajectoire du ballon. Dans l'étude de Macquet (2009), les résultats ont montré que le contenu de la catégorie «attentes » renvoyait à des informations sur les compétences ou capacités des adversaires et/ou des partenaires. Pour nous, ces informations étaient relativement stables, établies et renvoyaient plutôt à des connaissances. Comme Lenzen et al. (2009), nous avions regroupé au sein de la catégorie « attente » des événements susceptibles de se produire ou attendus dans la situation. Les attentes exprimées à travers les verbalisations du joueur de football, de hockey ou de handball concernaient les actions des partenaires et/ ou des adversaires susceptibles de se produire dans un futur proche. Une attente est une représentation occurrente relative à un moment et à une situation. Dans notre étude, la prise en compte des qualités et défauts des autres joueurs partenaires et/ou adversaires constituait un des contenus de la catégorie connaissance ce qui est concordant avec la proposition de Lenzen et al. (2009). La prise en compte des compétences des autres joueurs renvoie davantage à des représentations-types ou des connaissances. Ces connaissances construites sur les compétences d'autrui semblent influencer les attentes et les actions du joueur. La singularité de ces connaissances constituait alors une véritable ressource pour l'action du joueur.

Bien que l'interprétation du nombre d'occurrence des éléments impliqués dans l'activité décisionnelle doive être menée avec précaution dans une étude qualitative, nous observons tout de même une proportion plus grande de données pour les catégories «perception » ou « indices pertinents » comparativement aux autres catégories dans ces études qualitatives. Les joueurs experts en sports collectifs ne reportent pas toutes les informations qu'ils perçoivent pour prendre une décision mais convoquent uniquement des indices critiques ou significatifs de la situation («je vois le dernier défenseur entre nous deux, je le fixe pour passer»). Ces résultats confortent l'idée que les experts (sportifs ou non) en situation dynamique consacrent plus de temps à reconnaître la situation qu'à 
comparer diverses options possibles pour prendre une décision (Johnson \& Raab, 2003 ; Klein, 2008).

Les résultats obtenus relatifs aux contenus des éléments mobilisés dans et pour l'action confortent et se situent dans la continuité de ceux obtenus lors d'études antérieures (Lenzen et al., 2009 ; Macquet, 2009 ; Bossard et al., 2010). Cependant, c'est surtout dans la tentative de modélisation de la dynamique de l'activité individuelle collective que notre contribution peut être considérée comme originale. L'organisation collective semble également constituer un facteur qui affecte l'activité décisionnelle du joueur dans notre situation d'étude.

4. 2. Une tentative de modélisation de la dynamique de l'activité individuelle collective

En observant une certaine homogénéité des schémas mobilisés par les joueurs (13 schémas ont été mobilisés par différents joueurs d'une même équipe), nos résultats ouvrent vers l'étude de la manière dont se coordonnent les actions des membres d'un collectif sur la base d'une compréhension partagée de la situation (Cooke, Gorman, \& Winner, 2007). Dans la littérature consacrée aux activités collectives en situation sportive, une variété de cadres théoriques et méthodologiques, de définitions et de dénominations relatifs à la nature des éléments partagés coexistent (pour une revue voir Sève et al., 2009). Actuellement, les études sur l'activité collective s'organisent autour de deux axes. D'un côté, le partage est étudié en termes de mise en commun de connaissances stables mais opérationnelles entre chacun des membres d'une équipe: les «connaissances partagées » (Bourbousson \& Sève, sous presse). D'un autre côté, le partage des contenus cognitifs entre partenaires est plutôt étudié en termes d'informations contextuelles et d'actions qui concernent « l'ici et le maintenant » (e.g. Bourbousson, Poizat, Saury, \& Sève, 2008 ; Poizat, Bourbousson, Saury, \& Sève, 2009).

Dans le domaine du tennis de table, Poizat et al. (2009) ont par exemple caractérisé le partage d'informations au cours d'un match de double en mobilisant la notion de contexte partagé. Les résultats ont montré qu'entre deux pongistes, pourtant habitués à jouer ensemble, la compréhension partagée n'était pas permanente mais se construisait plutôt sur des points de recoupement ponctuels. A partir de l'étude de l'activité collective d'une équipe de basketball, Bourbousson et al. (2008) ont montré que les contenus cognitifs fluctuants se partageaient dynamiquement sur la base de recoupements à la fois ponctuels et locaux (i.e., entre certains joueurs). Les auteurs ont alors proposé que la compréhension partagée émerge de l'agencement d'îlots de compréhension locale, sans que les joueurs ne partagent nécessairement une 
compréhension commune sur le jeu. Notre étude, bien qu'elle traite de l'activité décisionnelle individuelle, s'inscrit dans la lignée des études précédentes, et ouvre des pistes relatives à l'étude du partage de contenus cognitifs plus stables tels que les schémas. En effet, au regard d'un ensemble d'études qui tendent à pointer le peu de partage à l'œuvre dans une activité collective viable, nos résultats (homogénéité des schémas observés entre les joueurs) invitent à bien distinguer le niveau d'appréhension des contenus cognitifs et de la compréhension partagée étudié.

Tout de même, les résultats de notre étude ont pointé une mobilisation privilégiée d'indices pertinents $(45,1 \%)$ au détriment des connaissances $(10,4 \%)$ par les joueurs de football. Aussi, au sein de cette catégorie «connaissances », peu d'unités significatives désignaient des connaissances stables alors que la plupart d'entre-elles concernaient des interprétations locales ou des jugements circonstanciés. En somme, en même temps que notre étude interroge le niveau d'appréhension du partage mobilisé dans les études récentes, elle confirme la fertilité de ce niveau d'analyse, et ouvre une réflexion sur les méthodes permettant d'identifier des contenus cognitifs stables, tels qu'une base partagée de connaissances. Nos résultats questionnent ainsi l'usage systématique d'entretiens d'autoconfrontation pour identifier la nature des éléments partagés par une équipe experte (ici, dans leur catégorie d'âge). L'utilisation complémentaire d'autres méthodes nous parait nécessaire pour comprendre de façon plus exhaustive les différentes formes de partage supportant la coordination.

Enfin, notre tentative de modélisation de la dynamique de l'activité à partir des coordinations préférentielles au sein d'une équipe met en exergue un couplage acteur contexte (partagé) mais aussi un couplage actuel - potentiel (par exemple, le schéma 8 induit 12 ou 14) ce qui suggère l'existence de scénarii partagés par des joueurs d'une même équipe.

4. 3. De l'analyse de l'activité à la modélisation d'un joueur virtuel

En décrivant la complexité de l'activité décisionnelle, les études « naturalistes » ont contribuées à renseigner sur la diversité et le contenu des éléments pris en compte pour décider en action dans les sports collectifs (Bossard et al., 2010 ; Lenzen et al., 2009 ; Macquet, 2009 ; Mouchet, \& Bouthier, 2006). Ces études qualitatives, en « capturant » les traits caractéristiques de l'activité décisionnelle experte en situation dynamique, aboutissent à des modélisations pertinentes du couple joueur-situation. Certaines études ont fourni des indications conceptuelles ou pragmatiques dans la perspective d'améliorer la prise de décision du joueur en sports collectifs (Lenzen et al., 2009 ; Macquet, 2009 ; Mouchet, 2005). Si ces modélisations issues de l'analyse de l'activité réelle ont été 
exploitées dans le cadre de propositions de situations de formation (Ria, Leblanc, Serres, \& Durand, 2006), leurs mobilisations pour les situations de simulation exploitant les techniques de la réalité virtuelle constituent une voie nouvelle et originale. Dans notre cas, la modélisation obtenue à partir de l'analyse de l'activité a été utilisée pour choisir et développer un modèle informatique de joueur de football virtuel pour enrichir ses comportements (pour une vue complète de la démarche voir Bossard et al., 2009).

Dans la perspective d'améliorer la crédibilité des comportements des joueurs virtuels dans CoPeFoot, deux modèles informatiques ont été nécessaires pour opérer un rapprochement avec le modèle RPD et la théorie des schémas: un modèle de raisonnement à partie de cas (Mille, 1999) et un modèle du contexte (Kokinov, 1997). La figure 3 présente la relation entre ces deux modèles.

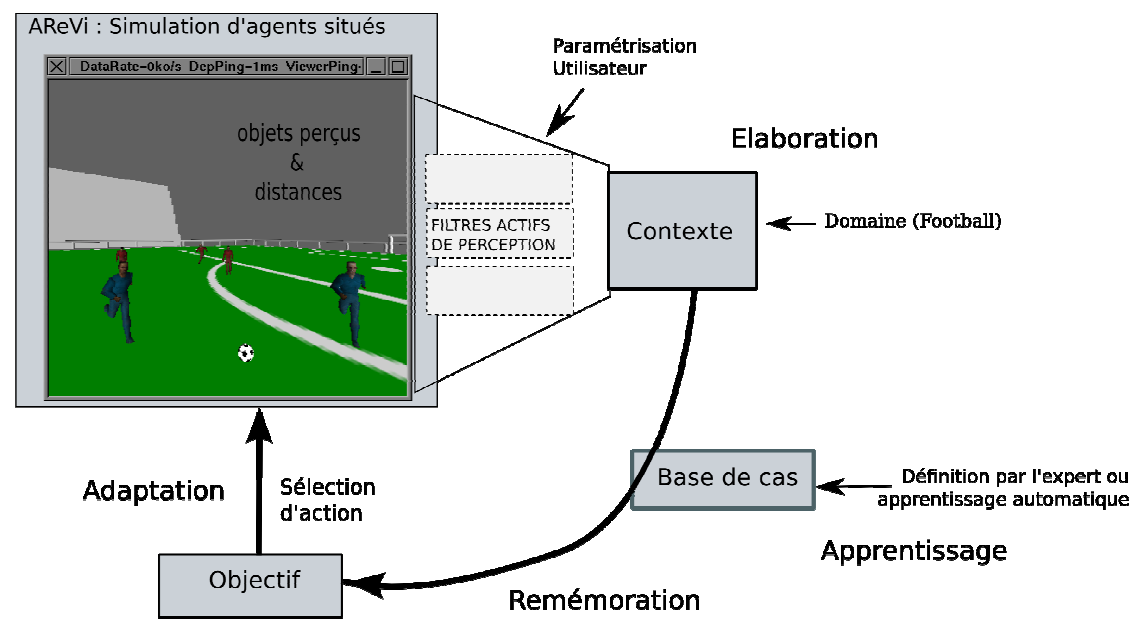

Figure 3. Le modèle RàPC et le modèle du contexte de CoPeFoot (Bénard, 2007).

Pour améliorer la crédibilité comportementale des joueurs virtuels, le modèle informatique a également été doté de possibilités d'évolution. Ces possibilités d'évolution peuvent recourir à l'aspect synchronique (schéma et ses constituants) et/ou diachronique (enchainement des schémas) de l'activité des joueurs réels.

Dans un premier temps, le modèle issu de l'analyse de l'activité peut directement être appliqué pour enrichir la base de cas et spécifier les perceptions des joueurs virtuels dans le contexte. Les schémas des joueurs constituent alors une base de données pour le chercheur en informatique. Ici, le modèle informatique du joueur virtuel est un calque du modèle humain. L'évolution du modèle est lié à un mécanisme d'apprentissage explicite ou par instructions. 
Une autre possibilité d'évolution consiste à enrichir la base de cas et le contexte à partir de l'usage. CoPeFoot est doté de possibilités d'interactions et l'utilisateur a la possibilité de cliquer sur des éléments qui sont significatifs pour lui. Ces « indices pertinents " sont représentés par des symboles comme la double flèche qui indique la distance par rapport à la cible ou entre deux adversaires, par des symboles qui soulignent la disponibilité ou le marquage, etc. Grâce à cet apport d'informations graphiques, l'utilisateur « explicite » les éléments significatifs dans l'action. Les renseignements fournis par l'utilisateur en cliquant sur ces éléments permettent alors de réviser les poids associés à chaque élément de contexte (voir l'algorithme dans Bénard et De Loor, 2007). L'évolution de l'environnement virtuel est ici liée à un mécanisme d'apprentissage par imitation dont les joueurs virtuels sont dotés.

Enfin, l'enrichissement du modèle est issu de la simulation. Le modèle informatique mémorise les nouveaux cas qui émergent directement des interactions entre utilisateur(s) et joueurs virtuels. "L'arbre des cas " (ou les scénarii privilégiés) se diversifie et s'affine pour offrir de nouvelles possibilités d'actions aux joueurs virtuels. L'évolution de l'environnement virtuel est liée à un mécanisme d'apprentissage implicite.

\section{Conclusion}

Cette étude sur l'activité décisionnelle que nous pouvons qualifier « d'individucentrée " a montré que l'activité décisionnelle de joueurs de football sous forte pression spatio-temporelle reposait en partie sur la perception des mêmes éléments significatifs en fonction de différentes phases de la situation de contre-attaque. Si certains schémas peuvent être considérés comme typiques de la contre-attaque en football, ils constituent une sorte de référentiel commun de jeu (Bourbousson et Sève, sous presse). II serait alors intéressant de passer d'une étude individu-centrée à une l'analyse de l'activité de l'équipe. À l'instar de Poizat, Bourbousson, Saury, \& Sève, C. (2009), il s'agirait d'identifier comment s'articulent ou se coordonnent les schémas de chacun des membres d'un collectif et d'identifier la nature du partage d'informations aux différents moments du cours d'action d'une équipe. De la même manière que pour l'activité individuelle, la modélisation de l'activité collective pourrait alors nourrir le choix ou la conception d'un modèle informatique et sa simulation à l'aide d'un dispositif exploitant les techniques de la réalité virtuelle. 


\section{Bibliographie}

Anderson, J. R., Matessa, M., \& Lebiere, C. (1997). ACT-R: A theory of higher level cognition and its relation to visual attention. Human Computer Interaction, 12, 439462.

Araùjo, D., Davids, K., \& Hristovski, R. (2006). The ecological dynamics of decisionmaking in sport. Psychology of Sport and Exercise, 7, 653-676.

Bardin, L. (2003). L'analyse de contenu. Paris: PUF (11ème Edition).

Bedny, G., Karwowski, W. \& Bedny, M. (2001). The principle of unity of cognition and behavior : implications of activity theory for the study of human work. International Journal of Cognitive Ergonomics, 5(4), 401-420.

Bénard, R. (2007). Raisonnement en contexte pour la simulation participative et l'étude des situations dynamiques collaboratives. Une application au sport collectif. Thèse de doctorat, Université de Bretagne Occidentale.

Bénard, R., \& De Loor, P. (2007). Context-Based Decision-Making for Virtual Soccer Players. In Proceedings of the international Conference on Context: Context' 07 (pp 2-15).

Bossard, C., \& Kermarrec, G. (sous presse). La prise de décision des joueurs de sports collectifs. Une revue de question en psychologie du sport. Science \& Motricité.

Bossard, C., Kermarrec, G., De Loor, P., Bénard, R., \& Tisseau, J. (2009). Sport, réalité virtuelle et conception de simulations participatives. Illustration dans le domaine du football avec le simulateur CoPeFoot. Intellectica, 2, 97-117.

Bossard, C., De Keukelaere, C., Cormier, J., Pasco, D., \& Kermarrec, G. (2010). L'activité décisionnelle en phase de contre-attaque en Hockey sur glace. @ctivités, 7 (2), 41-60. http://www.activites.org/

Bourbousson, J., Poizat, G., Saury, J., \& Sève, C. (2008). Caractérisation des modes de coordination interpersonnelle au sein d'une équipe de basket-ball. @ctivités, 5 (1), 21-39. http://www.activites.org/

Bourbousson, J., \& Sève, C. (sous presse). Construction/déconstruction du référentiel commun d'une équipe de basketball au cours d'un match. Impulsions.

Caron-Pargue, J., \& Caron, J. (1989). Processus psycholinguistiques et analyse des verbalisations dans une tâche cognitive. Archives de Psychologie, 57, 3-32. 
Chalandon, X. (2007). Conscience de la situation : invariants internes et invariants externes. Contributions de l'ergonomie cognitive et de l'ingénierie à la conception de systèmes d'aide à la gestion des environnements dynamiques. Thèse de Doctorat en Ergonomie. CNAM, Paris.

Cooke, N. J., Gorman, J. C., \& Winner, J. L. (2007). Team cognition. In F. Durso, R. Nickerson, S. Dumais, S. Lewandowsky \& T. Perfect (Eds.), Handbook of Applied Cognition (pp. 239-268). Wiley ( $2^{\text {nd }}$ Edition).

Federico, P. A. (1995). Expert and novice recognition of similar situations. Human Factors, 37(1), 105-122.

Fiore, S. M., \& Salas, E. (2006). Team cognition and expert teams: Developing insights from cross-disciplinary analysis of exceptional teams. International Journal of Sport and Exercise Psychology, 4 (4), 369-375.

Flin, R., Slaven, G., \& Stewart, K. (1996). Ermergency decision making in the offshore oil and gas industry. Human Factors, 38, 262-277.

Gansner, E. R., Koutsofios, E., North, S. C. \& Vo, K. P. (1993). A technique for drawing directed graphs. IEEE Transactions on Software Engineering, 19(3), 214-230.

Hoffman, R. R., \& Lintern, G. (2006). Eliciting and representing the knowledge of experts. In Ericsson, K.A., Charness, N., Hoffman, R.R. \& Feltovich, P.J. (Eds.): The Cambridge Handbook of Expertise and Expert Performance (pp. 203-222). Cambridge: Cambridge University Press.

Johnson, J. G., \& Raab, M. (2003). Take the first: Option generation and resulting choices. Organizational Behavior and Human Decision Processes, 91, 215-229.

Klein, G. (1997). The Recognition-Primed Decision (RPD) model: looking back, looking forward. In C.E. Zsambok \& G.A. Klein (Eds.), Naturalistic Decision Making (pp. 285-292). New Jersey: Lawrence Erlbaum Associates.

Klein, G. (2008). Naturalistic Decision Making. Human Factors, 50(3), 456-460.

Klein, G. A., \& Brezovic, C. P. (1986). Design engineers and the design process: Decision strategies and human factors literature. Proceedings of the Human Factors and Ergonomics Society $30^{\text {th }}$ Annual Meeting, 2, 771-775.

Klein, G., Ross, K.G., Moon, B.M., Klein, D. E., Hoffman, R.R., \& Hollnagel, E. (2003). Macrocognition. IEEE Intelligent Systems, 18(3), 81-85. 
Kokinov, B. (1997). A Dynamic Theory of Implicit Context. In Proceedings of the 2nd European Conference on Cognitive Science, Manchester.

Lenzen, B., Theunissen, C., \& Cloes, M. (2009). Situated Analysis of Team Handball Players'Decisions: An Exploratory Study. Journal of Teaching in Physical Education, 28, 54-74.

Lipshitz, R., Klein, G., Orasanu, J., \& Salas, E. (2001). Focus article: Taking stock of naturalistic decision making. Journal of Behavioral Decision Making, 14, 331-352.

Macquet, A. C. (2009). Recognition within the decision-making process: A case study of expert volleyball players. Journal of Applied Sport Psychology, 21, 64-79.

Mille, A. (1999). Tutorial RàPC, état de l'art du raisonnement à partir de cas, Plateforme AFIA'99.

Minsky, M. (1975). A framework for representing knowledge. In P. Winston (Ed.). The psychology of computer vision (pp. 211-277). New York: McGraw-Hill.

Mouchet, A. (2005). Modélisation de la complexité des décisions tactiques en rugby. EJournal de la Recherche sur I'Intervention en Éducation Physique et Sport, 7, 319.

Mouchet, A., \& Bouthier, D. (2006). Prendre en compte la subjectivité des joueurs de rugby pour optimiser l'intervention. STAPS, 72(2), 93-106.

Piaget, J. (1947). La psychologie de l'intelligence. Paris : Armand Colin.

Piegorsch, K. M., Watkins, K. W., Piegorsch, W. W., Reininger, B., Corwin, S. J., \& Valois, R. F. (2006). Ergonomic decision-making: A conceptual framework for experienced practitioners from backgrounds in industrial engineering and physical therapy. Applied Ergonomics, 37, 587-598.

Poizat, G., Bourbousson, J., Saury, J., \& Sève, C. (2009). Analysis of contextual information sharing during table tennis matches: An empirical study on coordination in sports. International Journal of Sport and Exercise Psychology, 7(4).

Randel, J. M., Pugh, H. L., \& Reed, S. K. (1996). Differences in expert and novice situation awareness in naturalistic decision making. International Journal of HumanComputer Studies, 45, 579-597.

Ria, L., Leblanc, S., Serres, G. et Durand, M. (2006). Recherche et formation en « analyse des pratiques » : un exemple d'articulation. Recherche et Formation, 51, 43-56. 
Ross, K. G., Shafer, J. L., \& Klein, G. (2006). Professional judgments and "naturalistic decision making". In Ericsson, K. A., Charness, N., Hoffman, R. R. \& Feltovich, P. J. (Eds.), The Cambridge Handbook of Expertise and Expert Performance (pp. 403-419). Cambridge: Cambridge University Press.

Rumelhart, D. E. (1980). Schemata: The building blocks of cognition. In R.J. Spiro, B.C. Bruce, \& W.F. Brewer (Eds.). Theoretical issues in reading and comprehension: Perspectives from cognitive psychology, linguistic, artificial intelligence, and education (pp. 33-58). Hillsdale, NJ: Erlbaum.

Schank, R. C., \& Abelson, R. P. (1977). Scripts, plans, goals, and understanding: An inquiry into human knowledge structures. Hillsdale, NJ: Lawrence Erlbaum.

Schmidt, R. A., \& Lee, T. D. (2005). Motor Control and Learning: A Behavioral Emphasis. Champaign: Human Kinetics.

Sève, C., Bourbousson, J., Poizat, G., \& Saury, J. (2009). Cognition et performance collective en sport. Intellectica, 52, 71-95.

Strauss, A., \& Corbin, J. (1998). Basics of Qualitative Research: Techniques and Procedures for Developing Grounded Theory. ( $2^{\text {nd }}$ ed.). Sage: Thousand Oaks.

Sugiyama, K., Tagawa, S. \& Toda, M. (1981). Methods for visual understanding of hierarchical systems. IEEE Transactions on Systems, Man, and Cybernetics, 11(2), 109- 125.

Vergnaud, G. (1990). La théorie des champs conceptuels. Recherches en Didactique des Mathématiques, 10(23), 133-170. 